\title{
Preface
}

\section{UCHIDA Keiichi*}

It has been seven years since we first proposed the academic framework of "cultural interaction studies," and five years have passed since we launched the Society for Cultural Interaction in East Asia.

The term, "cultural interaction (studies)" has come to be widely used recently in Japan and, in particular, overseas; conferences prefixed with the term are now held frequently. This indicates that the objective of the framework that we have been advocating has begun gradually penetrating the academic world. Yet it is a fact that when asked the fundamental question of "what exactly are cultural interaction studies?" we still have no definitive answer except for the basic understanding indicated below:

Research in cultural interaction originally meant-in the case of the history of Sino-Japanese interaction, for example - that the premise was a national research paradigm based on the unit of two countries. In the realms and fields of research, knowledge has been accumulated in individual narratives in the academic fields of language, philosophy, ethnic groups, religion, literature, or history; a methodology for the overall grasp of cultural interaction has been lacking. In contrast, the cultural interaction studies framework that we have put forward transcends nation states, ethnic groups, and even individual academic fields. Our model imagines East Asia as a defined entity with a complex cultural configuration. While focusing on the formation, transmission, contact, and change in cultures, cultural interaction studies is a new academic discipline that attempts to elucidate from a multi-perspective, integrated point of view the total state of cultural interaction.

One methodology we adopted for the establishment of this academic model was the "periphery approach." In this case, "periphery" refers not only to the regional concept but to the fact that there exists in academic and spatial fields a relationship between the periphery and center. Even in my own area of research, Chinese language studies, such a relationship can exist. Moreover, the "periphery" and "center" do not have a fixed relationship; it should be noted that a flexible relationship exists between the two concepts. Sometimes an entity can be considered the center and at other times, as the periphery. The relationship between periphery and center can also be mutually under-

\footnotetext{
* Vice President, Society for Cultural Interaction in East Asia, Kansai University
} 
stood as one between the individual and the general or between the unique and the universal. It is necessary to take a dialectal view of this relationship as "that and this," rather than "that or this."

Either way, this grand experiment has only just begun; in the future we must establish it as an academic model with concrete results through the process of regular debate and discussion.

Today scholarly research is becoming increasingly specialized. It should be pointed out that if we cavil over insignificant phenomena, the result will be an inability to hold far-reaching discussions of the whole. Even in terms of Chinese grammar, there is detailed research on extremely discreet phenomena, which doubtless constitutes progress in scholarship. On the other hand, with this approach it becomes impossible to construct a grammatical theory that encompasses the language as a whole. To begin with, the concrete definitions of sentences 文 (wen), subjects 主語 (zhuyu), and predicates 述語 $($ shuyu) are not explained, yet there are many corpora of itemized instances of the two uses of le/liao 了, definitions of the progressive zai 在, when $d e / d i$ 的 is or isn't necessary, or the differences between complements and serial verb modifiers (of course, elucidation of such individual items is also necessary). Yet the most fundamental question of the definition of language is overlooked. A view of language, or to put it on a larger scale, a world view, is lacking. Can we really call this progress or advancement in scholarship?

Furthermore, recently the methodologies of "interdisciplinarity" or "de-territorialization" have become something of a fad. These methodologies are actually quite commonsense approaches, but here, too, we should aim not to make too much of interdisciplinarity. We shouldn't forget that this is a fixed "interdisciplinarity" built on "specializations" and that interdisciplinarity and de-territorialization that lack a basis in specialization are untenable.

This is the fifth English-language journal published by the Society for Cultural Interaction in East Asia. It contains five articles, one book review, and two pieces introducing new research. We are certain that the journal will greatly contribute to the academic world. The sixth annual meeting of the Society for Cultural Interaction in East Asia will be held at Fudan University, Shanghai. One-hundred participants are expected to attend. As they say, "perseverance is strength." We hope the Society continues to grow in the future. 